\title{
FACTORES SOCIALES DE RIESGO Y PROTECCIÓN DEL SUICIDIO ADOLESCENTE
}

\section{Social risk factors and protection against adolescent suicide}

\author{
Héctor Rubén Bravo Andrade*, Mercedes Gabriela Orozco Solis**, \\ Norma Alicia Ruvalcaba Romero***, Cecilia Colunga Rodríguez****, \\ Mario Ángel González***** \\ Universidad de Guadalajara, Jalisco, México
}

\begin{abstract}
Resumen
Elfenómeno del suicidio en adolescentes involucra elementos sociales y contextuales que necesitan enriquecerse en la literatura especializada, por lo que el objetivo de la presente investigación fue conocer los factores sociales de riesgo y protección asociados a dicha problemática. Para ello se condujo un estudio cualitativo de tipo fenomenológico mediante grupos focales conformados por estudiantes, profesores y terapeutas, elegidos por medio de un muestreo por conveniencia. A través de la codificación de datos cualitativos emergieron como factores sociales de riesgo los medios de comunicación, especialmente el internet, así como el encontrarse envuelto en una situación de bullying. Por su parte, factores como el grupo de pares, la escuela y el contexto social se observaron como elementos que pueden representar tanto riesgo como protección. Se discute la necesidad de emprender acciones en favor del desarrollo de interacciones positivas desde un enfoque de promoción de la salud.
\end{abstract}

Palabras clave: suicidio, adolescentes, factores sociales de riesgo, factores sociales de protección, cualitativa.

\begin{abstract}
The phenomenon of adolescent suicide involves social and contextual issues that need to be enriched in a specialized literature, so the aim of the current research was to know the social risk and protective factors associated with this problem. A qualitative study was conducted through focus groups conformed by students, teachers and psychotherapists, chosen through a convenience sampling. Through qualitative data coding, emerged as social risk factors mass media, especially the internet, as well as been involved in a bullying situation. On the other hand,factors such as peer group, school and social context were identified as elements that can represent both risk and protective factors. It is discussed, the need of taking actions in order to develop positive interactions from a health promotion approach.
\end{abstract}

Keywords: suicide, adolescents, social risk factors, social protective factors, qualitative

\footnotetext{
* Psicólogo. Docente Universidad de Guadalajara. ruben.bravo.a@academicos.udg.mx

** Psicóloga. Universidad de Guadalajara.

*** Psicóloga. Universidad de Guadalajara.

**** Psicóloga. Universidad de Guadalajara.

***** Psicólogo. Universidad de Guadalajara.
} 


\section{INTRODUCCIÓN}

La adolescencia es, quizá de todas las etapas de la vida del ser humano, donde la influencia del contexto, más allá de la familia, cobra una significación mayúscula. Los adolescentes se encuentran ante el desafío de transición entre la niñez y la vida adulta (Bertolino, 2006), convirtiéndose en agentes que interactúan de manera directa con su medio (Taylor, 2004). Es preciso reconocer que la adolescencia misma varía según la sociedad en la que se encuentre (González, 2002), por lo que la forma en la que el adolescente afronte los cambios, inherentes a esta, se ve influida por su contexto particular (Fishman, 1995; Micucci, 2005).

Las principales tareas de desarrollo con las que se enfrenta el adolescente son la consolidación de identidad, el establecimiento de autonomía (Micucci, 2005), y la adquisición de destrezas que le permitan la integración en el mundo social, principalmente a través del grupo de pares (González, 2002; Leveton, 2005). Ahora bien, a partir del aumento en la autonomía, el adolescente se encuentra ante nuevas posibilidades de acción que configuran una serie de factores de riesgo y protección que vale la pena analizar de forma específica para este grupo poblacional (Cardozo y Dubini, 2005).

Las principales problemáticas de la adolescencia reportadas en la literatura, son el uso de alcohol y drogas, las relaciones sexuales sin protección (González, Unikel y Chávez, 2008), la indisciplina, los problemas de conducta, hechos violentos y trastornos de la conducta alimentaria (Cardoso y Dubini, 2005). Merece especial atención, de entre todas las problemáticas, el suicidio en el grupo adolescente, el cual representa la segunda causa de muerte en personas de entre 15 y 29 años, causando el $8.5 \%$ de muertes a nivel mundial (OMS, 2014). En México se ha encontrado un aumento del comportamiento suicida en la población de entre 15 y 25 años, llegando a constituir una tasa de 7.4 muertes por cada 100 mil adolescentes (INEGI, 2014).

Existe una amplia cobertura en lo que se refiere al estudio de la conducta suicida desde el ámbito individual (González-Forteza, Juárez, Montejo, Oseguera, Wagner y Jiménez, 2015; González-Forteza, Ramos, Caballero y Wagner, 2003; Hernández y
Lucio, 2010; Pérez-Amezcua, Rivera-Rivera, Atienzo, Castro, Leyva-López y Chávez-Ayala, 2010; Rivera y Andrade, 2008), y en menor grado en lo que respecta a la dimensión familiar (González-Forteza, Jimenez, Garfias y García, 2009; Runeson y Asbert, 2003; Valadez-Figueroa, Amezcua-Fernández, QuintanillaMontoya y González-Gallegos, 2005). Sin embargo, en lo tocante al impacto de los factores sociales vinculados al fenómeno suicida, más allá de las variables sociodemográficas (Borges, Medina-Mora, Orozco, Ouéda, Villatoro y Fleiz, 2009; Quintanilla y Varela, 2011), es más bien escaso el material disponible.

Los factores tanto de riesgo como de protección, no se limitan a las características individuales, ya sean estas de tipo biológico o psicológico, sino que trascienden la individualidad y se engarzan en contextos sociales (Rutter, 1999). Bajo este supuesto, el fenómeno del suicidio no se reduce al ámbito individual o familiar, sino que como Durkheim (2003) puso de manifiesto, involucra elementos de tipo social. En este sentido, la cabal comprensión del fenómeno del suicidio involucra ineludiblemente componentes psicosociales, históricos, económicos, culturales y contextuales (Corpas, 2011; González et al., 2008; OMS, 2014; Szasz, 1999).

Desde la dimensión social los principales factores de riesgo suicida para los adolescentes son, la presencia de conflictos y violencia en la comunidad (Farrell, Bolland \& Cockerham, 2015; OMS, 2014); el aislamiento social (Jiménez, Mondragón y GonzálezForteza, 2007); la agresión y la hostilidad (LucioGómez y Hernández-Cervantes, 2009); dificultades escolares y bullying (Cornell y Huang, 2016; SánchezSosa, Villareal-González, Musitu y Martínez, 2010); tener un amigo que ha cometido suicido (Bearman y Moody, 2004); y tener acercamiento con contenido que hace apología del suicidio a través de internet (Arroyo y Bertomeu, 2012; Hernández, 2009).

Por otra parte, los principales factores sociales de protección ante el riesgo de suicidio en adolescentes presentes en la literatura son, las redes sociales de apoyo y la cohesión comunitaria (Gómez y Kotliarenco, 2010; Kotliarenco, Cáceres y Fontanilla, 1997; Morgan, 2014; OMS; 2014; Rutter, 1999); ambientes escolares que favorezcan el enriquecimiento de los vínculos 
y oportunidades de participación significativa, y el establecimiento de límites claros y firmes (Cardozo y Dubini, 2005); y las relaciones interpersonales significativas con amigos y grupos de pares (OMS, 2014; Rutter, 2012).

Dada la importancia y la necesidad de enriquecer el cuerpo de investigaciones en relación al rostro social del fenómeno suicida, el objetivo del presente estudio fue conocer los factores sociales de riesgo y protección asociados al suicidio adolescente por parte de alumnos, profesores y terapeutas.

\section{MÉTODO}

\section{Diseño}

Se realizó un estudio cualitativo de tipo fenomenológico, considerando que se busca describir las experiencias desde la postura de los sujetos, partiendo de su propia percepción y marco de referencia (Martínez, 2001).

\section{Participantes}

La muestra incluyó 22 personas distribuidas en tres grupos focales: uno de alumnos de preparatoria, otro de profesores del mismo nivel educativo y uno más de terapeutas. Para la selección de los participantes se utilizó un muestreo por conveniencia, buscando incluir la misma proporción de hombres y mujeres dentro de cada grupo focal.

Para el grupo de alumnos, se incluyeron ocho estudiantes de una preparatoria pública del estado de Jalisco, México, los cuales podían ser de cualquier semestre o turno escolar. En el caso de los profesores, el grupo estuvo conformado por ocho docentes de la misma preparatoria, permitiendo que fueran de cualquier grado, asignatura o turno. Con respecto a los terapeutas, se contó con seis participantes, siendo seleccionados de manera independiente del enfoque terapéutico manejado y, a partir del criterio de inclusión de haber tenido contacto con el fenómeno del suicidio en cualquiera de sus expresiones. La conformación final de los grupos se describe en la Tabla 1.

Tabla 1

Características de los participantes

\begin{tabular}{|c|c|c|c|c|c|}
\hline \multicolumn{6}{|c|}{ Grupos focales } \\
\hline \multicolumn{2}{|c|}{ Alumnos } & \multicolumn{2}{|c|}{ Profesores } & \multicolumn{2}{|c|}{ Terapeutas } \\
\hline ID & Sexo & ID & Sexo & ID & Sexo \\
\hline GFA1 & Mujer & GFP1 & Mujer & GFT1 & Hombre \\
\hline GFA2 & Hombre & GFP2 & Hombre & GFT2 & Mujer \\
\hline GFA3 & Hombre & GFP3 & Hombre & GFT3 & Mujer \\
\hline GFA4 & Mujer & GFP4 & Mujer & GFT4 & Mujer \\
\hline GFA5 & Hombre & GFP5 & Mujer & GFT5 & Hombre \\
\hline GFA6 & Hombre & GFP6 & Mujer & GFT6 & Hombre \\
\hline GFA7 & Mujer & GFP7 & Hombre & & \\
\hline GFA8 & Hombre & GFP8 & Hombre & & \\
\hline
\end{tabular}

Nota: GFA: Grupo focal alumnos, GFP: Grupo focal profesores, GFT: Grupo focal terapeutas

\section{PROCEDIMIENTO DE RECOLECCIÓN DE DATOS}

La información recuperada en esta investigación se obtuvo por medio de grupos focales, llevando a cabo una sesión de entre 80 y 140 minutos con cada grupo. Las sesiones con alumnos y profesores se realizaron en el auditorio de la preparatoria, mientras que la reunión con los terapeutas se desarrolló en un espacio externo al plantel escolar. Todos los grupos focales fueron coordinados por el investigador principal, quien utilizó una guía de entrevista previamente elaborada (Tabla 2). Cabe señalar que todas las sesiones fueron audiograbadas, previa autorización de los participantes. 
Tabla 2

Preguntas guía de entrevista

\section{Preguntas}

¿Qué crees que le haría pensar a un adolescente en quitarse la vida?

¿Cuáles son las cosas que motivan a los adolescentes a vivir?

¿Cuál crees que sea el papel del medio social en el riesgo suicida?

¿De qué forma la escuela podría relacionarse con el riesgo suicida?

¿Qué podría hacer la escuela para empeorar esas situaciones?

¿De qué forma la escuela podría ayudar o evitar la presencia de riesgo suicida?

¿Cómo crees que manejan los chicos el riesgo suicida de alguno de sus pares?

¿De qué manera podrían los amigos ayudar a superar el riesgo de suicidio?

¿Qué pueden hacer los amigos del adolescente para empeorar esas situaciones?

¿Qué otros factores relacionas con el riesgo suicida en los adolescentes?

¿Hay algún otro comentario que quisieran agregar?

\section{ANÁLISIS DE DATOS}

La información obtenida a partir de los grupos focales fue transcrita en el procesador de texto Word y posteriormente analizada en el programa MAXQDA, en su versión 12.

El proceso de análisis cualitativo constó de tres etapas: En un primer momento, se realizó una lectura libre de los textos de cada grupo focal por separado, buscando identificar temas generales en su contenido. Posteriormente, continuando con el análisis independiente de cada grupo, se establecieron códigos que agruparan las aportaciones de los participantes, incluyendo los temas y subtemas emergentes de cada texto. Finalmente, se realizó el análisis de los tres grupos focales en conjunto, buscando conectar los temas elaborados de manera independiente en un cuerpo coherente de categorías globales. En esta etapa se tuvieron que fusionar o eliminar algunas de las tipologías planteadas durante los análisis realizados en fases anteriores (Taylor y Bogdan, 1987).

\section{Consideraciones éticas}

El proyecto de investigación fue avalado por el comité de ética de la preparatoria. La participación de todos los entrevistados fue voluntaria y confidencial, protegiendo su identidad en todo momento. En el caso de los estudiantes, se solicitó que presentaran el consentimiento informado completado por alguno de sus padres para poder participar.

\section{RESULTADOS}

En total se contó con 22 participantes, de los cuales 12 fueron hombres y 10 mujeres, distribuidos en tres grupos focales, uno de ocho alumnos, otro de seis terapeutas y un último de ocho profesores.

Del análisis cualitativo de los grupos focales emergieron dos temas generales: factores sociales únicamente de riesgo y factores sociales tanto de riesgo como de protección. En seguida se presentan los resultados de cada tema con las categorías que los componen (Figura 1), integrando fragmentos de las entrevistas grupales.

Figura 1. Factores sociales de riesgo y protección ante el suicidio en adolescentes

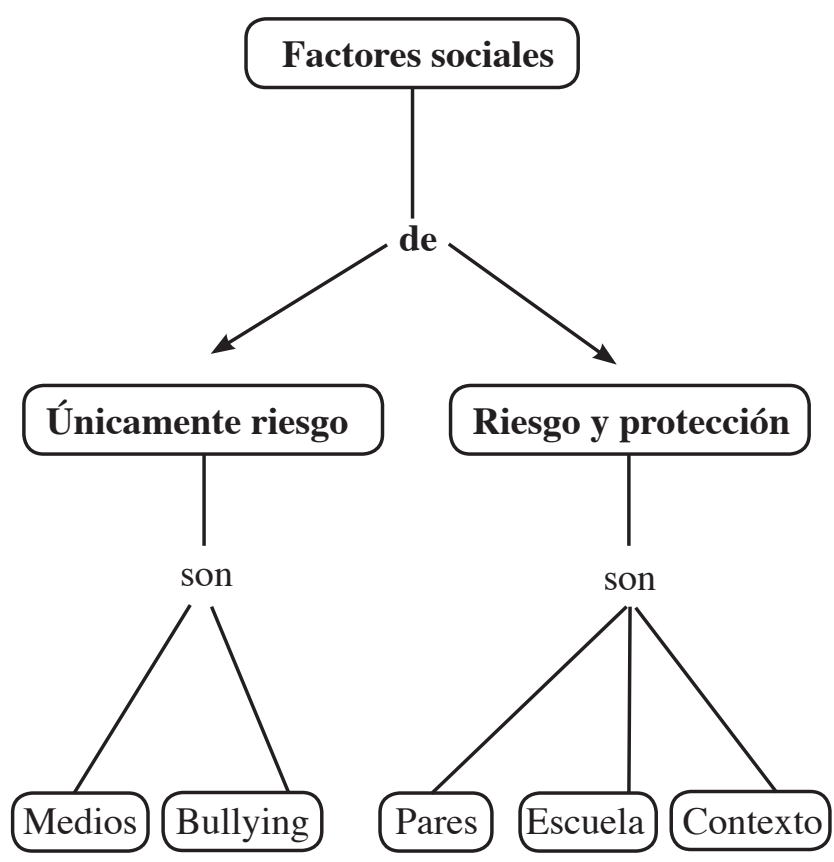




\section{Factores sociales de riesgo de suicidio en adolescentes}

En este tema se encontraron las categorías: medios de comunicación y bullying como potencial fuente de riesgo de conductas suicidas en estudiantes de preparatoria.

Medios de comunicación. Dentro de las aportaciones sobre esta categoría destacaron los estereotipos impuestos por las redes sociales, la exposición a la violencia y el desfase entre lo virtual y lo real, producto de las apariencias "maquilladas".

Para los alumnos una de las principales repercusiones de los medios de comunicación está dada por ciertos parámetros culturalmente establecidos que implican distinciones por sexo, y que al no ser alcanzados pueden producir malestar subjetivo.

también hay estereotipos para hombre, porque hay personas, como las mujeres "hay quiero ser delgada" también para los hombres, también se presionan mucho en obtener masa muscular (GFA4).

Los profesores destacaron que socialmente hay una exaltación de la violencia, sobre todo a partir de la llamada narco-cultura que impregna la vida cotidiana de manera inmediata.

siento que los medios de comunicación influyen demasiado en ellos, que ven demasiada violencia, asesinatos (GFP8).

Desde el grupo focal de los terapeutas se planteó que los medios de comunicación instauran estándares difícilmente alcanzables, lo cual arroja como consecuencia conflictos entre lo ideal y lo real.

el Facebook como plataforma de comunicación, modela perfiles de interacción y puede estar relacionado con la construcción de este nuevo imaginario de éxito que tiene que ver con esta cultura de ser feliz. O sea, no importa que no exista trabajo, o no importa que no tengamos los suficientes recursos, no importa que tu colonia de noche no tenga los recursos básicos... sé feliz. Entonces esta cultura de ser feliz, sin duda alguna que impacta, o sea, eso impacta en modelar ciertas formas de búsqueda de la inmediatez y de baja tolerancia a la frustración (GFT2).

Bulllying. Las situaciones de acoso escolar o bullying que pueda sufrir un adolescente en la escuela parecen surgir a partir de las diferencias o el no cumplimiento de las expectativas sociales, lo cual puede ocasionar sentimientos negativos que pueden orillarle a conductas suicidas.

Para los alumnos el bullying es un fenómeno que se refuerza de manera grupal, incluyendo burlas y ofensas, teniendo repercusiones en el estado anímico de los estudiantes.

hay veces que tratan de ser igual que otra persona y esa persona lo nota y ve y se empieza a burlar de él, empiezan a ofenderlo y todo su grupito también. Y pues él ya se ha de sentir un poco más solo, un poco más triste, le agarra la depresión (GFA1).

Los profesores hicieron mención sobre la manera en que los estereotipos influyen en las formas de interacción, y que el acoso direcciona la conducta del adolescente hacia lo socialmente aceptado, aunque sea en su perjuicio.

también fundamental, es la influencia de los propios compañeros, como que hay un prototipo de comportamiento a esa edad que si se sale empieza el bullying. Si ves a alguien a un muchacho serio y responsable los otros le están reclamando "tú por qué trajiste la tarea si nosotros no la trajimos", o sea ese tipo de presiones también orillan a determinados comportamientos que a lo mejor no los tenía pero ya están inmersos en un grupo que lo tiene (GFP3).

Entre los terapeutas surgió una aportación que plantea que el bullying no sólo se presenta de manera física, sino también a través de las redes sociales, en lo que se ha denominado cyberbullying. En estas vías alternas, no sólo se hace uso de la agresión directa, sino de exponer material sensible para la persona.

qué pasa cuando subes información de otra persona para evidenciarla, eso también es una forma de lastimar (GFT3). 


\section{Factores sociales de riesgo y protección}

En lo que respecta a este tema, emergieron las dimensiones pares, escuela y contexto social asociadas tanto al riesgo como a la protección ante el suicidio en adolescentes.

Pares. La principal noción que emerge en esta categoría es el peso que la presión social imprime en los adolescentes, pero al mismo tiempo pertenecer a un grupo genera satisfacción y la posibilidad de contención y derivación.

Desde el riesgo, en el grupo focal de los alumnos se encontraron dos ideas relacionadas en cuanto a la interacción con pares. Por un lado, se encuentra cierta falta de aceptación hacia las manifestaciones distintas a las mayorías. Lo cual lleva a la segunda cuestión; existe una gran presión social para sentirse incluidos en grupos de pares, lo cual, aunado al proceso de consolidación de la identidad, puede llevar a los adolescentes a realizar conductas de riesgo.

yo creo que los jóvenes ahorita piensan mucho en cómo caerle bien a los demás, cómo quedar bien con los demás. Si alguien se droga, se quiere meter a ese grupo social drogándose también, aunque sus ideales sean, no sé, hablando de la religión que vaya a misa, no sé, respete mucho las reglas sociales y por pertenecer a ese grupo se droga, o se avienta con los sicarios y eso, hacen, mata, tienen mucho dinero, carros y viejas a lo loco "ah pues yo también me quiero meter a eso". Y yo creo que es por quedar bien con los demás, porque no saben quiénes son, y como los aceptan en ese grupo hacen lo posible por quedar bien (GFA8).

Por otra parte, el grupo de pares puede fungir como un recurso protector al ser el primer medio de apoyo y contención ante el riesgo de suicidio, así como un conducto para dar parte a los adultos.

Pues a mí ya me pasó un caso así y lo que hicimos yo y mis amigas fue distraerla, o sea, la agarrábamos y nos íbamos corriendo, así sin sentido. Eso como que sí ayuda. Y también informamos a sus papás que estaba sensible y pues sus papás bien sorprendidos porque ellos no sabían nada (GFA4).
Entre los profesores se coincidió en que el grupo de iguales puede ser por una parte un riesgo, al mantener ideas distorsionadas sobre conductas a seguir.

tener ese vínculo (...) con un grupo de jóvenes que, a lo mejor se juntan en la anoche a hacer despapaye, pues puede ser causa de que piensen que todo lo que en su grupo se dice o se hace sea lo correcto (GFP2).

Y como protección al servir como un sistema secundario de acogimiento cuando se encuentran carencias en la familia.

el vínculo del joven con esa estructura familiar cualquiera que sea, pues no hay comunicación, no hay confianza, o sea se rompe esa unión, y busca otra unión que la sustituya; y la unión con sus amistades, novia o lo que sea, se convierte en algo primario para él (GFP2).

Por su parte, para los terapeutas, el que los adolescentes se encuentren en igualdad de condiciones puede significar que no se brinden el mejor apoyo posible entre sí.

más bien lo comunican con los pares y si esos pares también tienen problemas, pues ¿qué se van a decir? (GFT6).

Pero también los mismos pares pueden fungir como monitores entre sí de manera efectiva una vez que han sido capacitados.

me imagino una preparatoria que yo fuera compañero de alguien que tiene, que yo veo con la charla que nos dieron, que hay ciertos elementos que hacen probable que pueda cometer un acto suicida, pues yo "sabes que" casi, casi lo denuncio por decirlo así ¿no? con el psicólogo (GFT5).

Escuela. La principal cuestión surgida de esta categoría se refiere a la interacción profesor-alumno, y cómo desde esa relación se puede identificar el riesgo.

Para los alumnos una de las fallas por parte de la escuela, es que los profesores minimicen o pasen 
desapercibidos los problemas de los alumnos, incluso cuando ellos mismos lo refieren.

Me preguntó [la maestra] que qué me pasaba en la escuela, porque sabía que un niño me molestaba, ya le había dicho yo pero no le tomó importancia (GF8A).

Lo anterior debido a que identifican en el maestro a una figura de autoridad capaz de reconocer el riesgo suicida y tomar acciones para reducir el riesgo.

si un maestro se da cuenta que un niño está bajando su calificación o está muy serio siempre en las clases, o algo, hablar directamente con él o con alguien a quien él le hable, un amigo de él y buscar una solución. Tal vez, hablar con sus papás o una sesión en privado, hablar con toda la familia junta (GFA8).

De manera coincidente, en cuanto a la importancia de la relación entre maestros y alumnos, en el grupo de profesores se hizo mención de que en algunas ocasiones los maestros mismos juzgan o señalan a sus propios alumnos

Sí, a veces el mal trato de los maestros hacia con los alumnos también influye mucho, subajarlos (GFP1).

Pero por otro lado, pueden realizar labores de detección y derivación de aquellos casos que consideren de riesgo.

Yo pienso que primero hay que detectar. Hace rato hablábamos de comportamiento, de cambios de comportamiento o de aislamiento. O sea, detectar, "ah mira este alumno está muy apartado" o "este alumno no viene, no trabaja" hay que ver porqué. Y una vez detectando entonces canalizarlo (GFP3).

Entre los terapeutas se comentó que, a pesar de la importancia de espacios para la atención psicológica de los estudiantes, estos son insuficientes.

Yo creo que dentro de las escuelas hablando de, pues si estamos hablando dentro de las secundarias, a lo mejor primaria que realmente pocas escuelas le dan importancia como a un departamento psicopedagógico (GFT1).

\section{Contexto social}

En el grupo de alumnos no se encontraron manifestaciones en relación a esta dimensión desde el riesgo, pero sí se hizo mención de que las redes de apoyo hacen más llevadero el sufrimiento.

Sería como no lidiar tú solo con el problema, porque eso te causaría más daño (GFA1).

Los profesores pusieron de manifiesto que las realidades de los adolescentes no se restringen a su familia y la escuela, sino que el contexto más amplio en el que se desenvuelven puede, por una parte, favorecer un clima de violencia.

afuera son asaltados, les quitan los celulares en las colonias en donde ellos viven (GFP3).

Pero por otra parte, el contexto mismo puede tener un influjo positivo en ellos.

considero yo que la motivación proviene también del propio contexto donde se desenvuelven (GFP8).

Entre los terapeutas se destacó que las cuestiones macro sociales tienen un impacto en la vida particular de los adolescentes.

el contexto está determinado por una historia, está determinado por una cuestión económica, una cuestión política, una cuestión cultural y el contexto con esa densidad, digamos que en contextos de mayor precariedad de la vida nosotros podríamos ver mayores índices de ejercicios de la violencia, ya sea en las relaciones interpersonales o la violencia auto dirigida, en este caso como el suicidio (GFT2).

Y que la ampliación de su contexto, al incrementar las perspectivas, abre nuevas posibilidades de protección.

los niños cuando están pequeños pues es básicamente el contexto de la familia, está más 
tiempo, hablando en función del tiempo, pero conforme se van apartando, entonces a dónde están, es decir, este apoyo se tendría que buscar a donde se mueve yo creo que aunque esto suene paradójico la redes sociales son, también digo hay cosa muy positivas de información de instituciones que informan también a los chavos (GFT1).

\section{DISCUSIÓN}

El presente estudio tuvo como objetivo conocer los factores sociales de riesgo y protección asociados al suicidio adolescente por parte de alumnos, profesores y terapeutas. Las categorías de sentido emergidas de los grupos focales encuentran resonancia en la literatura especializada.

\section{Factores de riesgo}

Considerando que la identidad de los adolescentes se encuentra en proceso de consolidación, y que ese desarrollo encuentra eco en el contexto social (Micucci, 2005), los patrones estereotipados sobre cómo ser de tal o cuál forma, operan de manera acrítica en los adolescentes. Al respecto, Huerta, Morales, Reyes y Reyes (2016), encontraron que existe una relación entre el uso del internet, la identificación de roles normativos ante la sociedad y la autoevaluación personal. De lo anterior se desprende que la exposición reiterada a las redes sociales, podría cristalizar formas idealizadas de modelos a seguir; siendo la ideación suicida el desenlace a la frustración originada por compararse con tales parámetros inalcanzables.

Por otra parte, niños y adolescentes carecen de supervisión en el uso de internet, teniendo contacto con uno de los aspectos más negativos del uso de redes sociales: la exposición a contenidos violentos, que como consecuencia incrementan las interacciones agresivas en esta población (Arab y Díaz, 2015).

En relación al bullying, se encuentran en la literatura investigaciones que sustentan su relación tanto con la ideación como con el intento suicida en estudiantes mexicanos. Sánchez-Sosa et al. (2010) reportaron una relación directa significativa entre la victimización escolar y la ideación suicida, sobre todo si se encuentra junto con sintomatología depresiva y conducta alimentaria de riesgo. Por otra parte, los adolescentes que padecen acoso, disminuyen la interacción con sus pares, al grado del aislamiento, lo que les lleva a experimentar sentimientos de tristeza y soledad. En definitiva, la victimización o bullying guarda una asociación con el intento de suicidio en estudiantes del nivel medio superior (Valadez et al., 2011).

Con respecto al acoso presentado a través de medios electrónicos o cyberbullying, está documentado que se relaciona con la ideación y la conducta suicida en estudiantes, especialmente cuando carecen de indicadores de salud mental positiva (Brailovskaia, Teismann y Margraf, 2018).

\section{Factores de riesgo y protección}

Tanto los factores de riesgo como de protección son acumulativos, es decir, al sumarse potencializan su efecto (Kotliarenko, Cáceres y Fontanilla, 1997). Así también, ambos tipos de factores interactúan entre sí. En general, la forma de relacionarse es clara: incrementar factores de protección favorece la prevención, y su interacción con los de riesgo puede minimizarlos (González, Unikel y Chávez, 2008). Ahora bien, tal como sucedió con los resultados de la presente investigación en relación a las categorías pares, escuela y contexto, existen factores que pueden operar tanto como de riesgo como de protección, lo que hace más compleja su interpretación (Hidalgo, Villaseñor, Ramírez, Rasmussen, Nuño y Tapia, 2000). Por otro lado, es precisamente en la interrelación entre el riesgo y la protección que emergen los procesos resilientes (Morelato, 2011).

Como parte del proceso de individuación y consolidación de la identidad, el adolescente se independiza de su familia y se incorpora a la convivencia con su grupo de pares (Micucci, 2005; Medellín, Rivera, López, Kanán y Rodríguez-Orozco, 2012; Páramo, 2011). De este modo, se suelen establecer relaciones de amistad con otros jóvenes con quienes comparten gustos e intereses, así como una forma de pensar similar, lo cual puede reforzar las conductas desviadas que pudieran presentar (Micucci, 2005). En ese sentido, existe evidencia en la literatura que asegura que tener un amigo que ha cometido suicido, incrementa la posibilidad de ideación e intento suicida (Bearman y Moody, 2004; Clark, Robinson, Crengle, 
Fleming, Ameratunga, Denny, Bearinger, Sieving \& Saewyc 2011).

Por otra parte, la exclusión social supone una herida para la identidad inconclusa del adolescente que puede vivirse como insoportable, al grado de asociarse al riesgo de suicidio (Rivera y Andrade, 2008). Sin embargo, el sentirse aceptado por el grupo de pares supone una fortaleza para la dimensión social del adolescente al obtener un medio de apoyo y contención inmediato (Rutter, 2012; Valadez et al., 2011; Villalba, 2003). Por lo que establecer relaciones sociales significativas con amigos protege del riesgo suicida (Farrell et al., 2015; Morgan, 2014; OMS, 2004; Sánchez-Teruel y Robles-Bello, 2014).

Más aún, el grupo de iguales no sólo funge como un amortiguador del riesgo de suicidio, sino que en caso de aparición puede ser reconocido a través de ellos. Programas de intervención que han integrado al grupo de pares en la detección y derivación de los jóvenes con conductas suicidas, han mostrado ser más eficaces para identificar y reducir el riesgo suicida en las escuelas (Aseltine y DeMartino, 2004; Spirito y Hernandez-Cervantes, 2009).

En relación a la escuela, el fracaso por proveer un espacio de crecimiento adecuado y oportunidades de desarrollo a los estudiantes, propicia la vulnerabilidad hacia conductas riesgosas (Rutter, 2012). Más aún, si a esto se suma la falta de capacidad institucional y de los miembros de la comunidad educativa para tratar con situaciones próximas al riesgo; por lo que es necesaria la implementación de procesos autogestivos hacia la vinculación de las escuelas con los servicios de salud mental. Lo cual, permitiría llevar a cabo acciones respaldadas por instituciones profesionales en la detección e intervención de estudiantes en riesgo de suicidio (González-Forteza, Arana y Jiménez, 2008).

Sánchez-Sosa et al. (2010) reportan que existe una relación significativa entre la problemática de integración escolar y la ideación suicida. Por el contrario, los adolescentes perciben en la escuela un espacio seguro cuando se les brinda información y atención; que exista una estructura disciplinaria donde las reglas sean claras y justas; pero sobre todo cuando se establecen relaciones significativas maestro-alumno de apoyo basadas en el respeto y la calidez, aminorando las conductas de riesgo, incluidas la ideación y el intento de suicidio (Cornell y Huang, 2016; González y Rey, 2006). Así también, tomar en consideración la opinión de los alumnos aumenta el sentido de pertenencia, al mismo tiempo que posiciona al profesor como un agente de resiliencia (Arón y Milicic, 2011).

El desarrollo y las vivencias de las personas no ocurren de manera hermética, sino que responden a la interacción con distintos sistemas en diferentes niveles. De este modo, lo que sucede en cuestiones macroestructurales en cuanto a temas políticos, económicos y culturales, a través de la mediación de las comunidades, tiene un impacto en la vida individual de las personas (Bronfenbrenner, 1987). En esta línea de pensamiento, siendo el suicidio un fenómeno multifactorial (González et al., 2008) en el que participan las diferentes dimensiones antes mencionadas; González, Ramos, Mariño y Pérez (2002) abogan por una perspectiva ecológica para el estudio de la conducta suicida.

Bajo esta perspectiva, son considerados como factores de riesgo en la adolescencia, la carencia de redes sociales de apoyo en la comunidad (Medina, Herazo, Barrios, Rodelo y Salinas, 2017) y la falta de cohesión social (Rutter, 1999). Por su parte, GonzálezForteza, Borges, Gómez y Jiménez (1996) consideran a la inestabilidad social como un factor de riesgo para las conductas suicidas. Por otro lado, se ha establecido que la percepción de inevitabilidad de la violencia, así como la exposición a la misma, incrementan el riesgo de cometer intentos de suicidio, especialmente en adolescentes que viven en comunidades en situación de pobreza (Farrell et al., 2015).

Por el contrario, el apoyo social emocional, las redes sociales estructuradas y el sentido de pertenencia a un grupo o cultura (Sánchez-Teruel y Robles-Bello, 2014) son factores que protegen del riesgo de suicidio a los adolescentes. Después de todo, un contexto pobre no determina la calidad de vida de una persona (Fiorentino, 2008; Kotliarenco, 2000), sino que incluso, el apoyo social juega un papel importante en la felicidad (Gómez-Azcarate, Vera, Ávila, Musitu, Vega y Dorantes, 2014). 
A manera de integración, destaca el hecho de que escuela, pares y contexto funcionan tanto como factores de riesgo como de protección más no así los medios que sólo aparecen como factor de riesgo. Teniendo en cuenta que el monitoreo de los padres se considera como un recurso protector contra el riesgo de suicidio (González-Forteza y Andrade, 1995), convendría hacerlo extensivo al uso del internet en general y al uso redes sociales en particular; a través de la supervisión por parte de los padres mediante límites y consecuencias en el uso de dispositivos electrónicos en cuanto al tiempo y lugares acordados, así como a favorecer las interacciones cara a cara que permitan el establecimiento de vínculos afectivos (Arab y Díaz, 2015).

Sobresale de este modo que los factores de riesgo sean más que los de protección, por lo que uno de los temas pendientes es precisamente disminuir los primeros e incrementar los segundos. En esta dirección, se recomienda que los programas de intervención tomen en cuenta el fortalecimiento de relaciones significativas entre los adolescentes, esto es, favorecer interacciones positivas entre los estudiantes. Lo anterior plantea transitar de un modelo de prevención del riesgo a uno de promoción de la salud (Flores, 2007).

En esta misma línea, en relación a los profesores, como principales agentes de la institución escolar, se exhorta por un lado a dotarlos de herramientas para identificar y derivar signos de riesgo; y por otro, a poder generar espacios de convivencia positiva en las escuelas, de modo que favorezcan la salud y el bienestar de los docentes, así como implementar acciones que redunden en relaciones profesor-alumno más cálidas y significativas (Cornell \& Huang, 2016).

Desde el contexto más amplio, se sugiere una agenda que integre la vinculación entre instituciones educativas y de salud mental, también sustentada desde la lógica de la promoción, privilegiando intervenciones centradas en factores protectores que tomen en cuenta cuestiones culturales y ecológicas, resaltando las fortalezas y las características positivas de los adolescentes (Sánchez-Teruel y Robles-Bello, 2014).

La principal aportación de este estudio es presentar en su conjuntolosfactores socialesde riesgoy protección ante el suicidio en adolescentes, más relevantes para alumnos, profesores y terapeutas. En este sentido, se destacan como fortalezas de la presente investigación, la integración de las voces de distintos actores cercanos a la problemática, así como la profundización lograda mediante la metodología cualitativa. Por su parte, se reconocen como limitaciones la ausencia de la visión sobre la problemática de familias de estudiantes, de las instituciones encargadas de la salud mental, a través de algunos de sus representantes; así como de personas que directamente hayan experimentado alguna de las problemáticas suicidas. Es por esto que además de las recomendaciones planteadas anteriormente, se sugiere llevar a cabo estudios que incorporen las posturas de aquellos actores que no fueron considerados en la presente investigación.

\section{REFERENCIAS}

Álvarez-Gayou, J. (2007). Cómo hacer investigación cualitativa. Fundamentos y metodología. Barcelona: Paidós.

Arab, E., y Díaz, A. (2015). Impacto de las redes sociales e internet en la adolescencia: aspectos positivos y negativos. Recuperado de: Revista Médica Clínica las Condes, 26(1), 7-13. https:// doi.org/10.1016/j.rmclc.2014.12.001

Arón, A., y Milicic, N. (2011). Resiliencia y clima social en el contexto escolar. Psykhe, 4(1), 57-68. Recuperado de http://www.psykhe.cl/index.php/ psykhe/article/view/73/73

Arroyo, A., y Bertomeu, A. (2012). Métodos suicidas e internet. Revista Española Especialidad Medicina Legal, 38(4), 143-148. https://doi.org/10.1016/ j.reml.2012.07.003

Aseltine, R., \& DeMartino, R. (2004). An outcome evaluation of the SOS suicide prevention program. American Journal of Public Health, 94(3), 446451. Recuperado de https://www.ncbi.nlm.nih. gov/pmc/articles/PMC1448274/pdf/0940446.pdf

Bearman,P., \& Moody,J.(2004). Suicide and friendship among american adolescents. American Journal of Public Health, 94(1), 89-95. Recuperado de https://www.ncbi.nlm.nih.gov/pmc/articles/ PMC1449832/pdf/0940089.pdf 
Bertolino, B. (2006). Terapia orientada al cambio con adolescentes y jóvenes. Una nueva generación de procedimientos y prácticas respetuosos y eficaces. Barcelona: Paidós.

Borges, G., Medina-Mora, M., Orozco, R., Ouéda, C., Villatoro, J., y Fleiz, C. (2009). Distribución y determinantes sociodemográficos de la conducta suicida en México. Salud Mental, 32(5), 413-425. Recuperado de http://revistasaludmental.mx/ index.php/salud_mental/article/view/1309/1307

Brailovskaia, J., Teismann, T., \& Margraf, J. (2018). Cyberbullying, positive mental health and suicide ideation/behavior, Psychiatry Research, 267, 240-242. https://doi.org/10.1016/ j.psychres.2018.05.074

Bronfenbrenner, U. (1987). La ecología del desarrollo humano. Barcelona: Paidós.

Cardozo, G., y Dubini, P. (2005). Factores de riesgo y protección. Su incidencia en la conducta de los adolescentes. En Cardozo, G. (Coord). Adolescencia Promoción de salud y resiliencia (pp. 43-62). Córdoba: Brujas.

Clark, T., Robinson, E., Crengle, S., Fleming, T., Ameratunga, S., Denny, S., Bearinger, L., Sieving, R., \& Saewyc, E. (2011). Risk and protective factors for suicide attempt among indigenous Māori youth in New Zealand: The role of family connection. Journal of Aboriginal Health, 7(1), 1631. http://dx.doi.org/10.18357/ijih71201112350

Cornell, D., \& Huang, F. (2016). Authoritative school climate and high school student risk behavior: a cross-sectional multilevel analysis of student selfreports. Journal of Youth and Adolescence, 45(11), 2246-2259. https://doi.org/10.1007/s10964-0160424-3

Corpas, J. (2011). Aproximación social y cultural al fenómeno del suicidio. Comunidades étnicas amerindias. Gazeta de Antropología, 27(2). Recuperado de http://www.ugr.es/ pwlac/G27_ 33JoseManuel_Corpas_Nogales.html

Durkheim, E. (2003). El suicidio. México: Coyoacán.

Farrell, C., Bolland, J., \& Cockerham, C. (2015). The role of social support and social context on the incidence of attempted suicide among adolescents living in extremely impoverished communities.
Journal of Adolescent Health, 56(1), 59-65. https://doi.org/10.1016/j.jadohealth.2014.08.015

Fiorentino, M. (2008). La construcción de la resiliencia en el mejoramiento de la calidad de vida y la salud. Suma Psicológica, 15(1), 95-114. Recuperado de http://publicaciones.konradlorenz.edu.co/index. php/sumapsi/article/view/18/4

Fishman, H.C. (1995). Tratamiento de adolescentes con problemas. Un enfoque de terapia familiar. Barcelona: Paidós.

Flórez, L. (2007). Psicología social de la salud: promoción y prevención. Bogotá: El Manual moderno.

Hernández, Q. (2009). Suicidio adolescente y la internet. JOVENes. Revista de estudios sobre la juventud. julio-diciembre, 108-121. DOI: 10.13140/RG.2.1.4282.6320

Gómez-Azcarate, E., Vera, A., Ávila, M., Musitu, G., Vega, E., y Dorantes, G. (2014). Resiliencia y felicidad de adolescentes frente a la marginación urbana en México. Psicodebate, 14(1), 4568. Recuperado de https://www.palermo.edu/ cienciassociales/investigacion-y-publicaciones/ pdf/psicodebate/14/Psicodebate_N14_T1_03.pdf

Gómez, E., y Kotliarenco, MA. (2010). Resiliencia Familiar: un enfoque de investigación e intervención con familias multiproblemáticas. Revista de Psicología, 19(2), 103-131. Recuperado de http://repositorio.uchile.cl/bitstream/ handle/2250/129448/Resiliencia-familia-unenfoque-de-investigacion-e-intervencion-confamilias-multiproblematicas.pdf?sequence $=1 \&$ is Allowed=y

González, C., Ramos, L., Mariño, M., y Pérez, E. (2002). Vidas en riesgo: Conducta suicida en adolescentes mexicanos. Acta Psiquiátrica y Psicológica de América Latina, 48(1-4), 74-84.

González, C., Unikel, C., y Chávez, A. (2008). Acerca de la problemática suicida y la conducta alimentaria de riesgo en adolescentes estudiantes. En Andrade, P., Cañas, J., y Betancourt, D. (Comps.) Investigaciones psicosociales en adolescentes (pp. 111-134). Chiapas: UNICACH.

González, E. (2002). Desarrollo en la adolescencia (doce a dieciséis años) II.En González,E.(Coord.). Psicología del ciclo vital. Madrid: CCS. 
González-Forteza, C., y Andrade,P. (1995).La relación de los hijos con sus progenitores y sus recursos de apoyo: correlación con la sintomatología depresiva y de la ideación suicida en los adolescentes mexicanos. Salud Mental, 18(4), 4148. Recuperado de http://revistasaludmental.mx/ index.php/salud_mental/article/view/575/575

González-Forteza, C., Arana, D., y Jiménez, J. (2008). Problemática suicida en adolescentes y el contexto escolar: Vinculación autogestiva con los servicios de salud mental. Salud Mental, 31(1), 23-27. Recuperado de http://revistasaludmental.mx/ index.php/salud_mental/article/view/1206/1204

González-Forteza, C., Borges, G., Gómez, C., y Jiménez, A. (1996). Los problemas psicosociales y el suicidio en jóvenes. Estado actual y perspectivas. Salud Mental, 19(S1), 33-38. Recuperado de http://revistasaludmental.mx/ index.php/salud_mental/article/view/614/614

González-Forteza, C., Hermosillo, E., Vacío-Muro, M., Peralta, R., y Wagner, F. (2015). Depresión en adolescentes. Un problema oculto para la salud pública y la práctica clínica. Boletín Médico del Hospital Infantil de México, 72(2), 149-155. https://doi.org/10.1016/j.bmhimx.2015.05.006

González-Forteza, C., Jiménez, JA., Garfias, N., y García,F. (2009). Problemática suicida en familias fragmentadas. JOVENes, 32, 79-89. Recuperado de https://amsmx.files.wordpress.com/2014/02/ gonzc3a1lez-forteza-c-jimc3a9nez-j-a-garfias-ngarcc3ada-f-2014-problemc3a1tica-suicida-enfamilias-fragmentadas.pdf

González-Forteza, C., Juárez, C., Montejo, L., Oseguera, G., Wagner, F., y Jiménez, A. (2015). Ideación suicida y su asociación con drogas, depresión e impulsividad en una muestra representativa de estudiantes de secundaria del estado de Campeche, México. Acta Universitaria, 25(2), 29-34. Recuperado de http://www.redalyc. org/articulo.oa?id=41648310005

González-Forteza, C., Ramos, L., Caballero, MA., y Wagner, F. (2003). Correlatos psicosociales de depresión, ideación e intento suicida en adolescentes mexicanos. Psicothema, 15(4), 524532. Recuperado de http://www.psicothema.es/ pdf/1102.pdf
González, M., y Rey, L. (2006). La escuela y los amigos: factores que pueden proteger a los adolescentes del uso de sustancias activas. Enseñanza e Investigación en Psicología, 11(1), 23-37. Recuperado de http://www.redalyc.org/ html/292/29211102/

Hernández, Q., y Lucio, E. (2010). Modelo de evaluación del riesgo suicida adolescente basado en la exposición a eventos estresantes y configuraciones de personalidad. En Eguiluz, L., Córdova, M., Rosales, J. (comp.). Ante el suicidio. Su comprensión y tratamiento (117-136). México: Pax.

Huerta, V., Morales, K., Reyes, K., y Reyes, C. (2016). Construcción de la identidad: Estereotipos de género mediados a través de internet. PsicoEducativa: Reflexiones y Propuestas, 2(4), 45-57.

Instituto Nacional de Estadística y Geografía. (2014). Estadísticas a propósito del día mundial para la prevención del suicidio. Recuperado de http:// www.inegi.org.mx/inegi/contenidos/espanol/ prensa/Contenidos/estadisticas/2014/suicidio0. pdf

Jiménez, A., Mondragón, L., y González-Forteza, C. (2007). Self-esteem, depressive symptomatology, and suicidal ideation in adolescents: results of three studies. Salud Mental, 30(5), 20-26. Recuperado de http://revistasaludmental.mx/index.php/salud_ mental/article/view/1186/1184

Kotliarenco, M., Cáceres, I., y Fontanilla, M. (1997). Estado de arte en resiliencia. Washington DC: Organización Panamericana de la Salud. Recuperado de http://www1.paho.org/hq/ dmdocuments/2009/Resil6x9.pdf

Leveton,E.(2005).Terapiafamiliarpara el adolescente en crisis. México. Pax.

Lucio-Gómez, E., y Hernández-Cervantes, Q. (2009). Personalidad y riesgo suicida en adolescentes estudiantes. Revista Médica del Instituto Mexicano del Seguro Social, 47(1), 33-40. Recuperado de http://www.medigraphic.com/pdfs/imss/im-2009/ ims091g.pdf

Martínez, M. (2001). Comportamiento humano. México: Trillas. 
Medellin, M., Rivera, M., López, J., Kanán, G., y Rodríguez-Orozco, A. (2012). Funcionamiento familiar y su relación con las redes de apoyo social en una muestra de Morelia, México. Salud Mental, 35(2), 147-154. Recuperado de http:// revistasaludmental.mx/index.php/salud_mental/ article/view/1467/1465

Medina, A., Herazo, M., Barrios, A., Rodelo, Y., y Salinas, L. (2017). Factores psicosociales asociados a la conducta suicida en adolescentes. Avances en Psicología, 25(1), 49-57. Recuperado de http://revistas.unife.edu.pe/index.php/ avancesenpsicologia/article/view/133/100

Micucci, J. (2005). El adolescente en la terapia familiar. Cómo romper el ciclo del conflicto y el control. Buenos Aires: Amorrortu.

Morelato, G. (2011). Resiliencia en el maltrato infantil: aportes para la comprensión de factores desde un modelo ecológico. Revista de Psicología 29(2), 203-224.

Morgan, A. (2014). Revisar el Modelo de los Activos: aclaración de ideas y términos. Global Health Promotion, 21(2), 91-94. Recuperado de http:// journals.sagepub.com/doi/pdf/10.1177/17579759 14537152

OMS. (2014). Prevención del suicido: un imperativo global. Washington, DC: OMS. Recuperado de http://apps.who.int/iris/bitstream/handle/10665/1 36083/9789275318508_spa.pdf;jsessionid=ADF A4C154964A73DBFA157DEC9BAC7B0?seque nce $=1$

Páramo, M. (2011). Factores de riesgo y factores de protección en la adolescencia: Análisis de contenido a través de grupos de discusión. Terapia Psicológica, 29(1), 85-95. http://dx.doi. org/10.4067/S0718-48082011000100009

Pérez-Amezcua, B., Rivera-Rivera, L., Atienzo, E., Castro, F., Leyva-Lopez, A., y Chávez-Ayala, R. (2010). Prevalencia y factores asociados a la ideación e intento suicida en adolescentes de educación media superior de la República mexicana. Salud Pública de México, 52(4), 324333. Recuperado de http://www.scielo.org.mx/ pdf/spm/v52n4/v52n4a08.pdf

Quintanilla, R., y Varela, C. (2011). Suicidio en menores de 18 años: estudio descriptivo de casos en la Zona Metropolitana de Guadalajara. En Chávez, A., Betancourt, L. (Comp.) Abordajes multidisciplinares sobre el suicidio en México. Memorias del IV Congreso Internacional de Prevención de Suicidio: México: Asociación Mexicana de Suicidología, A.C.

Rivera, M., y Andrade, P. (2008). Conductas autodestructivas e intento suicida en los adolescentes.EnAndrade,P.,Cañas, J.,Betancourt, D. (Comps.) Investigaciones psicosociales en adolescentes (pp. 89-110). Chiapas: UNICACH.

Runeson, B., \& Asberg, M. (2003). Family history of suicide among suicide victims. American Journal of Psychiatry, 160(8), 1525-1526. https://doi. org/10.1176/appi.ajp.160.8.1525

Rutter, M. (1999). Resilience concepts and findings: implications for family therapy. Journal of Family Therapy, 21(2), 119-144. https://doi. org/10.1111/1467-6427.00108

- (2012). Resilience: Casual pathways and social ecology. En Ungar, M. (Edit.). The social ecology of resilience. A handbook of theory and practice (pp. 33-42). New York: Springer.

Sánchez-Sosa, J., Villareal-González., M., Musitu, G., y Martínez, B. (2010). Ideación suicida en adolescentes: Un análisis psicosocial. Intervención Psicosocial, 19(3), 279-287. Recuperado de http:// www.redalyc.org/pdf/1798/179817507008.pdf

Sánchez-Teruel, D., y Robles-Bello, M. (2014). Factores protectores que promueven la resiliencia ante el suicidio en adolescentes y jóvenes. Papeles del psicólogo, 35(2), 181-192. Recuperado de http://www.papelesdelpsicologo.es/pdf/2438.pdf

Spirito, A., \& Hernandez-Cervantes, Q. (2009). Prevention of suicidal behaviorduring adolescence. En DiClemente, R., Santelli, J., Crosby, R. (Ed). Adolescent health. Understanding and preventing risk behaviors (233-247). USA: Wiley.

Swick, K. \& Williams, R. (2006). An Analysis of Bronfenbrenner's Bio-Ecological Perspective for Early Childhood Educators: Implications for Working with Families Experiencing Stress. Early Childhood Education Journal 33(5), 371378. DOI: 10.1007/s10643-006-0078-y

Szasz, T. (1999). Libertad fatal. Ética y política del suicidio. Barcelona: Paidós. 
Taylor, J. (2004). Salutogenesis as a framework for child protection: literature review. Journal of advanced nursing, 45(6), 633-643. https://doi. org/10.1046/j.1365-2648.2003.02954.x

Taylor, S., \& Bogdan, R. (1987). Introducción a los métodos cualitativos de investigación. Barcelona: Paidós.

Valadez-Figueroa, I., Amezcua-Fernández, R., Quintanilla-Montoya, R., y González-Gallegos, N. (2005). Familia e intento suicida en el adolescente de educación media superior. Archivos en Medicina Familiar, 7(3), 69-78. Recuperado de http://www.medigraphic.com/pdfs/medfam/amf2005/amf053b.pdf
Valadez, I., Amezcua, R., González, N., Montes, R., y Vargas, V. (2011). Maltrato entre iguales e intento suicida en sujetos adolescentes escolarizados. Revista Latinoamericana de Ciencias Sociales, Niñez y Juventud, 2(9), 783-796. Recuperado de http://www.scielo.org.co/pdf/rlcs/v9n2/v9n2a20. pdf

Villalba,C.(2003).El concepto de resiliencia individual y familiar. Aplicaciones en la intervención social. Intervención psicosocial, 12(3), 283299. Recuperado de http://www.redalyc.org/ pdf/1798/179818049003.pdf

Fecha de recepción: 30 de octubre 2018

Fecha de aceptación: 19 de noviembre 2018 